\title{
MALADIE DE KIMURA: À PROPOS D'UN CAS
}

\author{
N. DIB, A. BENHAMMOU, N. NAZIH, L. ESSAKALI, M. KZADRI \\ SERVICE D'ORL ET DE CHIRURGIE CERVICO-FACIALE, \\ HÔPITAL DES SPÉCIALITÉS, CHU RABAT-SALÉ, RABAT, MAROC
}

\begin{abstract}
RESUME
Introduction : La maladie de Kimura ou lymphogranulome éosinophile est une pathologie inflammatoire chronique très rare, d'étiologie inconnue. Nous rapportons un cas de maladie de Kimura à localisation parotidienne et à travers une revue de la littérature, rappelons les principales caractéristiques cliniques, paracliniques, thérapeutiques et évolutives de cette pathologie.

Matériel et méthodes : Patient de 17 ans qui a consulté devant l'apparition d'une tuméfaction de la région parotidienne gauche évoluant depuis un an et sans paralysie faciale.

Résultats : L'échographie cervico-parotidienne a révélé une parotide gauche hypertrophiée siège de multiples nodules hypoéchogènes. La tomodensitométrie cervico-faciale a mis en évidence une glande parotide gauche augmentée de taille et de structure hétérogène nodulaire sans adénopathies cervicales. La cytopontion était non concluante. Le patient a bénéficié d'une parotidectomie exofaciale gauche et l'étude anatomopathologique de la pièce opératoire revenue en faveur de la maladie de Kimura. Les suites opératoires étaient simples. Le recul est d'un an sans récidive.

Discussion : La Maladie de Kimura se caractérise cliniquement par des nodules sous cutanés de localisation cervicofaciale, une augmentation du volume des glandes salivaires et des adénopathies satellites. Son diagnostic est histologique reposant sur l'identification d'une hyperplasie follicullaire avec des abcès à polynucléaires éosinophiles. Le traitement est chirurgical le plus souvent mais dans certains cas la corticothérapie est proposée. Ce diagnostic doit cependant rester à l'esprit devant toute masse cervico-faciale.
\end{abstract}

Mots-clés : Maladie de Kimura, parotide, chirurgie.

Introduction : Kimura's disease (lymphogranuloma with hypereosinophilia) is a very rare form of chronic inflammatory disorder. We report a new case of parotid location of this pathology, and through a review of the literature, we expose the different aspects clinic, paraclinic, therapeutic and evolutive of this pathology.

Observation : 17 years old patient, who has consulted for a firm parotid tumefaction occurred since one year, without facial paralysis.

Results : The sonography showed a hypertrophy of the parotid with multiple hypoechogenic nodules. The CTscan conclude to the same aspect, without cervical lymph nodes. The cytology ponction was non contributive.

The diagnosis was confirmed by the histopathology, after exofacial parotidectomy. The evolution was favourable, without recidive after one year.

Discussion : Kimura's disease is characterized by multiple subcutaneous nodules in the cervico-facial area, a hypertrophy of the salivary glands and cervical lymph nodes. The diagnosis is histological, and repose on identification of follicular hyperplasia, and abscess with neutrophils. The treatment is surgical, but some authors propose corticotherapy.

Keywords : Kimura's disease, parotid gland, surgery

\section{INTRODUCTION}

La maladie de Kimura ou lymphogranulome éosinophile est une affection très rare. II s'agit d'une pathologie inflammatoire chronique d'étiologie inconnue. La maladie est endémique en Orient et sporadique dans le reste du monde (1).

Nous rapportons un cas de maladie de Kimura à localisation parotidienne et à travers une revue de la littérature, rappelons les principales caractéristiques cliniques, paracliniques, thérapeutiques et évolutives de cette pathologie.

\section{OBSERVATION CLINIQUE}

Notre patient était un jeune homme de 17ans, sans antécédents pathologiques notables, qui a consulté pour une tuméfaction de la région parotidienne gauche, évoluant depuis 1 an et demi, augmentant progressivement de volume, sans douleur ni épisodes infectieux ou inflammatoires avec l'absence de trismus et d'asymétrie faciale. L'examen clinique trouvait un patient en bon état général, apyrétique, avec des conjonctives normocolorées.

A l'inspection on notait une tuméfaction de la région parotidienne gauche sans signes inflammatoire en regard.

A la palpation, cette tuméfaction siégeait au niveau du 
lobe superficiel de la parotide gauche, de consistance ferme, polylobée, mal limitée, non douloureuse, non battante, mobile par rapport aux plans superficiel et profond, mesurant environ $4 \mathrm{~cm}$ de grand diamètre.

Par contre la palpation de la région parotidienne controlatérale était sans particularités ainsi que celle des deux régions sous maxillaires.

Les aires ganglionnaires cervicales étaient libres.

L'examen de la cavité buccale et de l'oropharynx ne montrait pas d'écoulement purulent ou hématique par l'orifice d'abouchement du canal de Sténon ni de bombement de la paroi postéro-latérale de l'oropharynx.

L'examen ORL complet et l'examen général étaient normaux.

Une échographie cervico-parotidienne a été demandée et a mis en évidence une glande parotide gauche hypertrophiée, d'échostructure hétérogène, siège de plusieurs nodules hypoéchogènes, homogènes, d'allure inflammatoire, le plus volumineux est polaire supérieur mesurant $12 \times 6 \mathrm{~mm}$

Une tomodensitométrie cervico-faciale a montré une glande parotide gauche augmentée de taille, de contours irréguliers (fig.1), infiltrant le tissu graisseux sous cutané en dehors (fig. 2), siège de multiples nodules hypodenses prenant le contraste de façon homogène sans visualisation d'image lithiasique ni de lyse osseuse.

Cependant, le scanner n'a pas objectivé d'adénopathie cervicale.

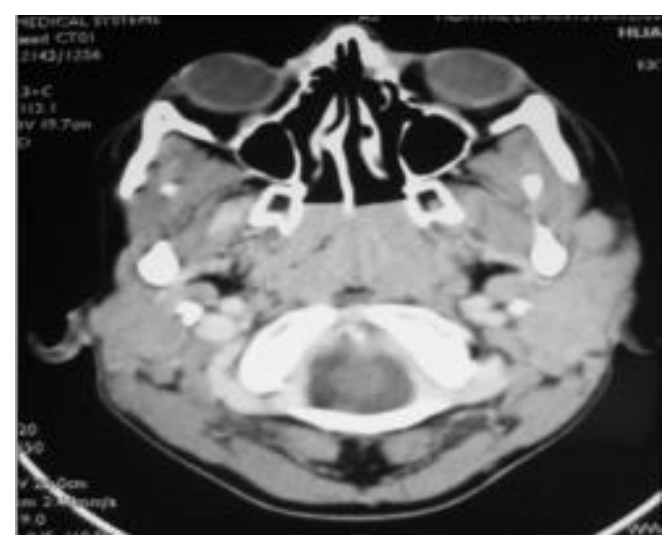

Fig. 1: Tomodensitométrie en coupes axiales montrant une glande parotide gauche augmentée de taille et de structure hétérogène

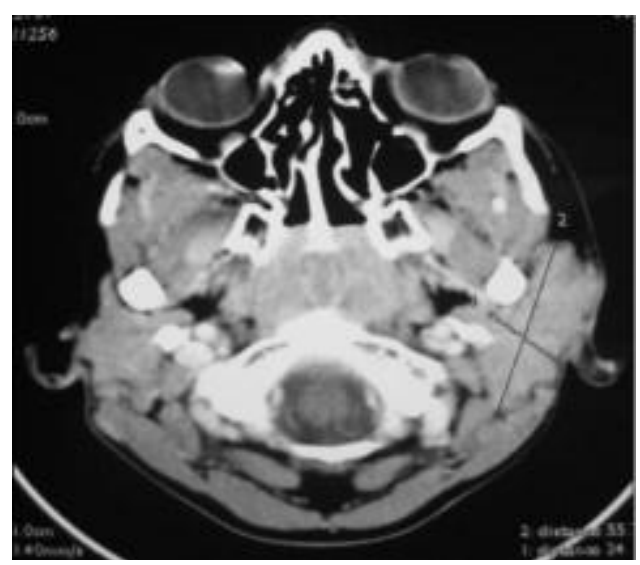

Fig. 2: Tomodensitométrie en coupes axiales montrant l'infiltration de tissu graisseux sous cutané en dehors.

La cytoponction de la glande parotide gauche a révélé une inflammatoin à cellules polymorphes avec l'absence de cellules suspectes.

Le bilan biologique était normal à part une hyperéosinophilie à 1584 éléments/mm3.

Nous avons décidé de réaliser chez ce patient une parotidectomie exploratrice gauche avec un examen extemporané.

L'exploration chirurgicale a trouvé une parotide gauche siège de multiples nodules d'allure inflammatoire.

L'examen extemporané était en faveur d'une réaction inflammatoire non spécifique faite essentiellement de lymphocytes, sans signe de malignité.

Le patient a bénéficie d'une parotidectomie exofaciale gauche. Les suites opératoires étaient simples.

L'étude anatomopathologique de la pièce a objectivé un parenchyme parotidien détruit par de nombreux follicules lymphoïdes à centre clair (fig. 3) avec une infiltration des polynucléaires éosinophiles réalisant des micro-abcès (fig. 4), ce qui était en faveur de la maladie de Kimura.

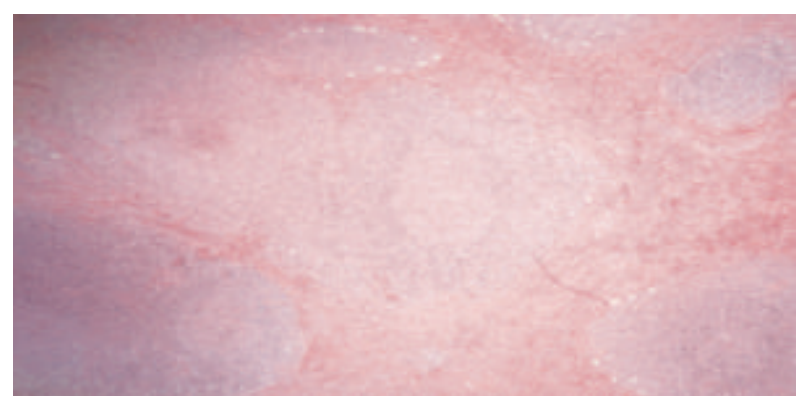

Fig. 3: Hyperplasie des follicules lymphoïdes à centre clair avec des plages importantes de fibrose 


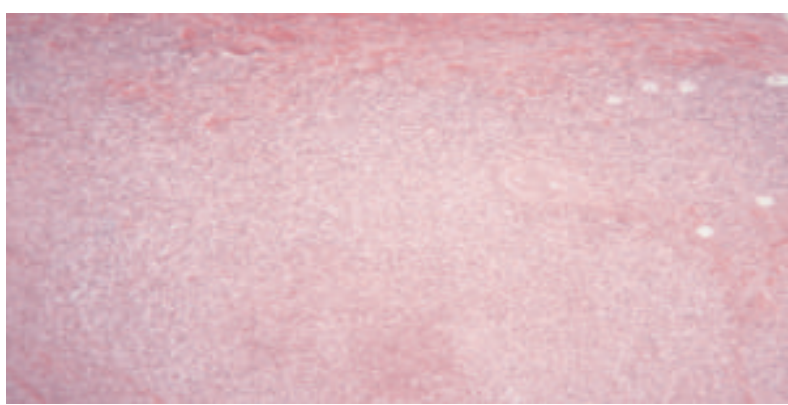

Fig. 4: Infiltration des polynucléaires éosinophiles dans les zones interfolliculaires et perivasculaires réalisant des micro-abcès à PN éosinophiles avec prolifération capillaire et veinulaire constituée par cellules endothéliales normales

Devant ce diagnostic histologique le bilan a été complété par un dosage des immunoglobulines sériques $E(\lg E)$ et d'une protéinurie de $24 \mathrm{~h}$ à la recherche d'un syndrome néphrotique, qui se sont révélées normales.

Le malade se présente régulièrement à la consultation avec un recul d'un an sans récidive.

\section{DISCUSSION}

La maladie de Kimura a été décrite la 1ère fois en 1937 dans la littérature chinoise par Kimm HT mais la description histologique définitive a été publiée par Kimura en 1948 et la maladie porte désormais son nom $(1,2)$.

La maladie est endémique en Orient (Chine et Japon) et sporadique dans le reste du monde. C'est une pathologie très rare : 200 cas rapportés dans le monde depuis sa description. On note une prédominance masculine avec un sex-ratio de 3,5 . Le pic d'âge se situe dans la 3ème décennie $(1,3)$.

II s'agit d'une pathologie inflammatoire chronique d'étiologie inconnue (2).

Elle est considérée par certains comme une réaction proliférative à un stimulus antigénique inconnu (4). Aucun réarrangement clonal des gènes n'a été retrouvé.

L'hypothèse qu'une stimulation antigénique prolongée peut conduire à une prolifération clonale lymphoïde a été émise. Les mastocytes producteurs d'interleukine 4 et 5 joueraient un rôle important dans la physiologie de la maladie de Kimura, en régulant la synthèse des immunoglobulines $\mathrm{E}$ ( $\lg \mathrm{E})$ et promouvant l'infiltration des polynucléaires éosinophiles $(1,5)$.

Cliniquement la maladie est caractérisée par l'apparition lente et progressive d'un ou plusieurs nodules sous cutanés, fermes ou mous, assez bien limités, de taille variable (3 à $10 \mathrm{~cm}$ ), recouverts d'une peau normale, non adhérents aux plans profonds, indolores, parfois prurigineux $(1,4)$.

La localisation préférentielle est cervico-faciale comme l'illustre notre observation (région péri-auriculaire, joues, faces latérales du cou et creux sus-claviculaires) $(3,4)$. Les localisations épitrochléenne, axillaire, inguinale et poplitée sont décrites (1).

Les orbites, oreilles, cuir chevelu et avant bras sont rarement atteints.

Exceptionnellement les lésions peuvent siéger au niveau des muqueuses (3).

Les atteintes extracutanées sont fréquentes comportent une atteinte des glandes salivaires en particulier la glande parotide; comme le cas de notre patient; et la glande sous maxillaire sous forme d'une augmentation du volume de ces dernières dans $70-100 \%$ des cas.

Les adénopathies locorégionales non inflammatoires sont quasi-constantes.

L'atteinte rénale est présente dans $50 \%$ des cas et se manifeste par une protéinurie ou un syndrome néphrotique lié souvent à une glomérulonéphrite extramembraneuse (6). Par ailleurs, les signes généraux sont absents (4).

Sur le plan biologique une hyperéosinophilie sanguine est quasiment retrouvée comme le cas de notre malade et il existe fréquemment une hyperimmunoglobulinémie $\mathrm{E}$.

Systématiquement, il faut rechercher une protéinurie qui sera témoin d'un syndrome néphrotique $(1,7)$.

L'examen anatomopathologique des lésions montre une hyperplasie du tissu lymphoïde avec des centres germinatifs florides, une infiltration modérée à massive par des polynucléaires éosinophiles dans les zones interfolliculaires et périvasculaires formant parfois des « abcès » à polynucléaires éosinophiles et une prolifération capillaire et veinulaire avec des vaisseaux réguliers à bordure constituée par des cellules endothéliales normales ou cuboïdales. Les follicules lymphoïdes sont composés de lymphocytes $T$ en périphérie et de lymphocytes $B$ dans les centres germinatifs. Des dépôts d'lgE sont fréquemment observés dans les follicules $(4,8)$. La fibrose est plus marquée dans les nodules sous cutanés et les glandes salivaires que dans les adénopathies (1).

Le diagnostic différentiel de la maladie de Kimura se fait principalement avec l'hyperplasie angiofolliculaire avec éosinophilie. C'est une pathologie d'Occident, elle touche les femmes d'âge moyen ( 3 à 5 décennies).

Elle se distingue de la maladie de Kimura par des nodules sous cutanés recouverts d'une peau érythémateuse ou pigmentée, la rareté des adénopathies et l'absence de l'augmentation des $\lg E(1,4)$. L'étude anatomopathologique montre le même aspect que la maladie de Kimura mais avec une prolifération vasculaire faite des cellules endothéliales turgescentes avec un cytoplasme vacuolisé dites « histiocytoïdes ». Ainsi l'hyperplasie angiofolliculaire avec éosinophilie correspondrait à une prolifération vasculaire bénigne $(4,8)$.

Le traitement de la maladie de Kimura n'est pas codifié. Différentes modalités thérapeutiques ont été proposées. Le traitement chirurgical est indiqué en 1ère intention comme c'était le cas de notre patient, il consiste à réaliser une exérèse large et profonde afin d'éviter les récidives 
qui sont fréquentes (1).

La corticothérapie constitue de loin le traitement médical le plus fréquemment utilisé. Elle est indiquée dans les formes profuses ou inaccessibles à la chirurgie ou systématiquement en cas d'atteinte rénale. Elle est prescrite à la dose initiale de 0,5 à $1 \mathrm{mg} / \mathrm{kg} / \mathrm{j}$ de prednisone avec une dégression lente sur au moins 6 mois avec une bonne efficacité. Cependant les rechutes sont possibles à l'arrêt de traitement.

Une chimiothérapie systémique à base de 5-fluoro-uracil et l'azathioprine a été utilisée en association à une corticothérapie générale mais sans que son utilité soit formellement prouvée (4).

La radiothérapie locale peut être proposé dans les formes réfractaires aux corticoïdes ou lorsque la chirurgie est impossible. La dose totale efficace est comprise entre 25 et 30 Gy.

Actuellement d'autres thérapeutiques sont en cours d'essai sans conclusion formelle. En effet, la Cetirizine a été utilisée et a pu induire chez un patient corticostéroïdes dépendant une rémission complète après 2 mois de traitement (1). Dans un cas de maladie de Kimura, l'interféron alpha a été prescrit en association à une corticothérapie générale mais n'a pas permis d'éviter une rechute lors de la diminution celle-ci (4).

L'évolution est marquée par des remissions spontanées mais les récidives sont notées chez plus de $25 \%$ de patients traités par chirurgie seule. Le pronostic est bon et aucune transformation maligne n'est rapportée $(1,2)$.

\section{CONCLUSION}

La maladie de Kimura est une affection rare, qui touche généralement les hommes japonais, âgés de 20 à 40 ans. Elle se caractérise par des nodules sous cutanés prédominant au niveau de la tête et du cou. Son diagnostic est histologique. Le traitement n'est pas codifié mais souvent on a recours à la chirurgie seule comme le cas de notre patient. Cependant, pour certains cas l'indication d'une corticothérapie générale s'avère nécessaire. Le pronostic de la maladie reste bon.

\section{REFERENCES}

1 - Laroche C, Bletry O. La maladie de kimura. Encéclopédie Orphanet. Février 2005

2 - V. Camlels, F. Lafitte, M. Sahli-Amor, R. Guillevin et al. Masses cervicales récidivantes en rapport avec une maladie de Kimura. Journal de neuroradiologie. 2005 ; 32( 4$): 287-8$

3 - Ray V. ; Boisseau-Garsaud A.-M. ; Hillion G. Maladie de Kimura à localisation palatine chez un Antillais. Revue de médecine interne.2003; 24(4):253-256 4- Kimura et hyperplasie angiolymphoïde avec éosinophilie. Thérapeutique dermatologique, Médecine-Sciences Flammarion @ 2001
5- Kimura Y, Pawankar R et al. Mas cells and t clls in Kmura's disease express increased levls of interleukin-4, interleukin-5, eotaxin and RANTES. Clin Exp Allergy 2002; 32:1787-93

6- Shetty AK, Beaty MW, McGuirt WF JR, Woods CR, Givner LB. Kimura's disease: a diagnostic challenge. Pediatrics 2002; 110: 39-45

7- Chen H, Thompson LD t al. Kimura disease: a clinicopathologic study of 21 cases. Am J Surg Pathol. 2004; 28:505-13

8- El sayed F, Dhaybi R et al. Angiolymphoid hyperplasia with eosinophilia: efficacy of isotretinoin. Head and Face Medecine 2006,2:32,5p 